TRANSACTIONS OF THE

AMERICAN MATHEMATICAL SOCIETY

Volume 357, Number 5, Pages 1859-1875

S 0002-9947(04)03615-3

Article electronically published on September 23, 2004

\title{
GRAPHS OF ZEROS OF ANALYTIC FAMILIES
}

\author{
ALEXANDER BRUDNYI
}

\begin{abstract}
Let $\mathcal{F}:=\left\{f_{\lambda}\right\}$ be a family of holomorphic functions in a domain $D \subset \mathbb{C}$ depending holomorphically on $\lambda \in U \subset \mathbb{C}^{n}$. We study the distribution of zeros of $\left\{f_{\lambda}\right\}$ in a subdomain $R \subset \subset D$ whose boundary is a closed nonsingular analytic curve. As an application, we obtain several results about distributions of zeros of families of generalized exponential polynomials and displacement maps related to certain ODE's.
\end{abstract}

\section{INTRODUCTION}

1.1. This paper is devoted to the problem of the distribution of zeros for families of univariate analytic functions depending analytically on a multivariate parameter. Such problems arise in several diverse areas of Modern Analysis, including Dynamical Systems (started by the classical works of Poincaré, Dulac and Bautin related to the so-called second part of Hilbert's $16^{\text {th }}$ problem [I] ), Transcendental Number Theory (going back to the classical works of Gelfond, Mahler and Siegel related to Hilbert's $7^{\text {th }}$ problem [B]) and Approximation Theory (Bernstein-Markov-Remez' type inequalities for analytic functions see e.g. [Br1], $[\mathrm{RY}]$ ). In $[\mathrm{Br} 2$ we studied this problem in the case of analytic families appearing as displacement maps of certain planar polynomial vector fields. The main result of that paper was derived from a new distributional inequality obtained by methods of Pluripotential Theory. In contrast, the main purpose of the present paper is to study some geometrical properties of the distributions of zeros.

Let $\mathcal{F}:=\left\{f_{\lambda} \in \mathcal{O}(D): \lambda \in U\right\}$ be a non-zero family of functions holomorphic in a planar domain $D \subset \mathbb{C}$ and depending holomorphically on $\lambda$ varying in a domain $U \subset \mathbb{C}^{n}$. The central set of $\mathcal{F}$ in $U$ is a closed complex analytic subset defined by

$$
C_{\mathcal{F}}:=\left\{\lambda \in U: f_{\lambda}=0\right\} .
$$

Given a subdomain $R$ compactly embedded in $D$ (written, $R \subset \subset D$ ), one introduces a function $\mathcal{N}_{\mathcal{F}, R}: U \backslash C_{\mathcal{F}} \rightarrow \mathbb{Z}_{+}$counting the number of zeros (with their multiplicities) of $f_{\lambda}$ in the closure $\bar{R}$, that is to say,

$$
\mathcal{N}_{\mathcal{F}, R}(\lambda):=\#\left\{z \in \bar{R}: f_{\lambda}(z)=0\right\} .
$$

This function is upper semicontinuous and bounded in $V \backslash C_{\mathcal{F}}$ for any subdomain $V \subset \subset U$ (see e.g. $[\mathrm{Br} 2]$ ). Finding the geometric properties of $\mathcal{N}_{\mathcal{F}, R}$ is the main objective of this paper. As an example of the results obtained we mention a rather

Received by the editors August 7, 2003.

2000 Mathematics Subject Classification. Primary 30C15; Secondary 34C07, 05C99.

Key words and phrases. Graph of zeros, exponential polynomial, displacement map.

The author's research was supported in part by NSERC.

(C)2004 American Mathematical Society 
unexpected "intermediate value theorem" asserting that for generic families $\mathcal{F}$ the function $\mathcal{N}_{\mathcal{F}, R}$ assumes on $V \backslash C_{\mathcal{F}}$ all (integer) values between its infimum and supremum.

We now introduce some basic definitions and related auxiliary results that will be used throughout the paper. Our main notion is related to a result which, in turn, requires the definition of subanalytic sets. For convenience of the reader we recall

Definition 1.1. Let $M \subset \mathbb{R}^{N}$ be an open set. $X \subset M$ is said to be semianalytic in $M$ if for each $x \in M$ there exist its open neighbourhood $O_{x} \subset M$ and a finite family of real analytic functions $f_{i j}: O_{x} \rightarrow \mathbb{R}$ such that

$$
X \cap O_{x}=\bigcup_{i=1}^{p} \bigcap_{j=1}^{q} X_{i j},
$$

where $X_{i j}=\left\{f_{i j}(x)=0\right\}$ or $\left\{f_{i j}(x)>0\right\}$.

A subset $X$ of $M$ is subanalytic if, locally, $X$ is a projection of a relatively compact semianalytic set.

We refer to $[\mathrm{BM}]$ and $[\mathrm{H}$, and references therein, for the main results for basic properties of subanalytic sets used in the present paper.

Now (and below), let $\mathcal{F}, U, R$ and $V$ be the above introduced objects. Suppose that

$$
\text { for any } \xi \in D \text { there is a } \lambda \in U \text { such that } f_{\lambda}(\xi) \neq 0 \text {; }
$$

Let us introduce the set $S \subset V$ as the projection on $V$ of the analytic set

$$
\widetilde{S}=\left\{(z, \lambda) \in \Gamma \times V: f_{\lambda}(z)=0\right\} .
$$

Proposition 1.2 ([Br2] Lemma 4.2]). (1) $C_{\mathcal{F}} \cap V \subset S$.

(2) $S \subset V$ is a closed subanalytic subset of real dimension at most $2 n-1$.

(3) $V \backslash S$ is the disjoint union of a finite number of open sets $Z_{i}$ such that $\mathcal{N}_{\mathcal{F}, R}$ equals $i$ on $Z_{i}$.

In accordance with the properties of subanalytic sets, $Z_{i}$, its closure $\overline{Z_{i}}$, boundary $\partial Z_{i}:=\overline{Z_{i}} \backslash Z_{i}$, and its connected components are all subanalytic sets in $V$.

Now we are in a position to introduce our main notion.

Definition 1.3. The graph of zeros $\mathcal{G}_{\mathcal{F}, R}(V)$ is formed by the set of integers $\mathcal{N}_{\mathcal{F}, R}(V \backslash S)$ regarded as its vertices and the set of edges $(i, j)$, where $i, j \in$ $\mathcal{N}_{\mathcal{F}, R}(V \backslash S)$ are such that they satisfy the condition

$$
\operatorname{dim}_{\mathbb{R}}\left(\partial Z_{i} \cap \partial Z_{j}\right)=2 n-1 .
$$

The following instance illustrates this definition.

Example 1.4. (1) Let $\mathcal{F}:=\left\{f_{\lambda}: \lambda \in \mathbb{C}^{2 k}\right\}$, where

$$
f_{\lambda}(z)=\left(\lambda_{1} z+\lambda_{2}\right)^{n_{1}} \cdots\left(\lambda_{2 k-1} z+\lambda_{2 k}\right)^{n_{k}}, \quad n_{s} \in \mathbb{N}, \quad 1 \leq s \leq k .
$$

As the set $V \subset \mathbb{C}^{2 k}$ we choose the open Euclidean ball of radius $r$ centered at 0 (written, $V=B_{c}(0, r)$ ). In turn, choose $R$ to be the open disk $\mathbb{D}_{\rho} \subset \mathbb{C}$ of radius $\rho$ 
and center 0 . It is readily seen that the corresponding set $S \subset B_{c}(0, r)$ is the union of $k$ hypersurfaces given by

$$
\left|\lambda_{2 s-1}\right| \rho=\left|\lambda_{2 s}\right|, \quad 1 \leq s \leq k .
$$

Then the graph $\mathcal{G}_{\mathcal{F}, R}(V)$ can be described in this case as follows.

Let $\left\{e_{s}: 1 \leq s \leq k\right\}$ be the canonical basis of $\mathbb{R}^{k}$, and $P$ the parallelepiped formed by the vectors $n_{s} e_{s}, 1 \leq s \leq k$. Introduce a function $h$ defined on the set of vertices $\mathcal{V}$ of $P$ by $h(x):=\sum_{s=1}^{k} x_{s}, x \in \mathcal{V}$. If $h$ separates the points of $\mathcal{V}$, then the image of $h$ is the set of vertices for the graph $\mathcal{G}_{\mathcal{F}, R}(V)$. Moreover, a pair $(h(x), h(y))$ is an edge of this graph if $x, y$ are joined by a 1-dimensional edge of $P$. The figure below presents the case of $k=3, n_{1}=1, n_{2}=2$ and $n_{3}=4$.

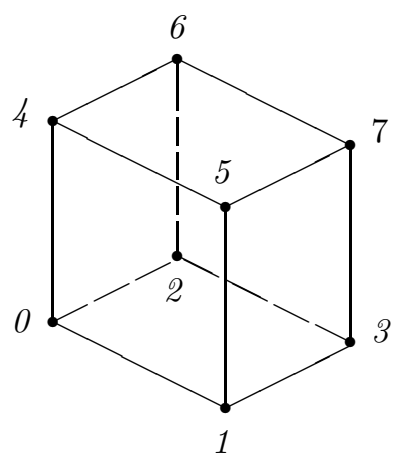

If $h$ does not separate the points of $\mathcal{V}$ we say that $x \sim y, x, y \in \mathcal{V}$, if $h(x)=h(y)$. This relation partitions the set $\mathcal{V}$ into equivalence classes $\{[x]: x \in \mathcal{V}\}$. Now the set of vertices of $\mathcal{G}_{\mathcal{F}, R}(V)$ is the image of $h$. Moreover, a pair $(h(x), h(y))$ is an edge of this graph if $h(x) \neq h(y)$ and there are $x^{\prime} \in[x]$ and $y^{\prime} \in[y]$ that are joined by a 1-dimensional edge of $P$. We leave the details to the reader.

(2) Now let $\mathcal{F}:=\left\{f_{\lambda}: \lambda \in \mathbb{C}\right\}$, where

$$
f_{\lambda}(z)=\left(\lambda+z^{n}\right) \cdot\left(1+\lambda z^{m}\right), \quad n \neq m .
$$

Choose $V:=\mathbb{D}_{2}$ and $R:=\mathbb{D}_{1}$. Then the corresponding set $S \subset \mathbb{D}_{2}$ is the unit circle $S^{1}=\partial \mathbb{D}_{1}$, and the graph $\mathcal{G}_{\mathcal{F}, R}(V)$ in this case has the form

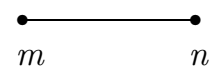

It is worth noting that in this case $\sup _{\mathbb{D}_{2}} \mathcal{N}_{\mathcal{F}, R}=n+m$, and it is attained on $S^{1}$.

Even these simple examples show that the graph of zeros may be of a complex combinatorial structure. Strikingly, for "generic" families $\mathcal{F}$ this graph has a linear structure described by the picture

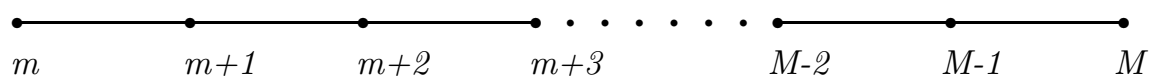

where $m$ and $M$ are infimum and supremum of $\mathcal{N}_{\mathcal{F}, R}$ on the set $V \backslash C_{\mathcal{F}}$. Adopting the terminology of Lie group theory, we refer to this as a graph of A-type. Our main results describe certain conditions on $\mathcal{F}$ under which the corresponding graph of zeros is of $A$-type. Remark 1.6 below shows that a small perturbation of an arbitrary family $\mathcal{F}$ gives a new family satisfying the conditions of our main theorem. 
To formulate these results it will be useful to introduce several additional notions.

We say that a simple closed curve $\Gamma \subset \mathbb{C}$ is periodic, if there exists an integer $n \geq 2$ such that $z \cdot e^{\frac{2 \pi i}{n}} \in \Gamma$ for any $z \in \Gamma$.

Now let a curve $\Gamma \subset \mathbb{C}$ be contained in the set $D$ from the definition of the family $\mathcal{F}$. We say that $\mathcal{F}=\left\{f_{\lambda}\right\}$ has no multiple zeros on $\Gamma$ if for any $w \in \Gamma$ the analytic set

$$
\left\{\lambda \in U: f_{\lambda}(w)=\frac{d f_{\lambda}}{d z}(w)=0\right\}
$$

has complex codimension at least 2 .

Now let $T(\eta), \eta=\left(\eta_{1}, \eta_{2}, \eta_{3}\right) \in \mathbb{C}^{3}$, be an operator defined on $g \in \mathcal{O}(D)$ by

$$
(T(\eta) g)(z):=g\left(\eta_{1} z+\eta_{2}\right)+\eta_{3}
$$

here $\eta_{1} z+\eta_{2} \in D$. This operator is well-defined for $z$ belonging to any compact $K \subset D$ and $\eta$ sufficiently close to the point $(1,0,0)$.

We say that the family $\mathcal{F}$ is locally stable with respect to the family $T$ on a compact $K \subset D$ if for any $\lambda \in U$ there is an open neigbourhood $O_{\lambda}$ of the point $(1,0,0)$ in $\mathbb{C}^{3}$ such that for $\eta \in O_{\lambda}$,

$$
\left(T(\eta) f_{\lambda}\right)(z)=f_{\nu}(z)
$$

with $\nu$ continuously depending on $\lambda$ and $\eta$, and $z \in K$.

Similar definitions will be used for the families of operators $T_{1}\left(\eta_{1}, \eta_{2}\right):=$ $T\left(\eta_{1}, 0, \eta_{2}\right), T_{2}\left(\eta_{1}, \eta_{2}\right):=T\left(\eta_{1}, \eta_{2}, 0\right)$, and $T_{3}(\eta):=T(\eta, 0,0)$.

Now we are in a position to formulate our main results. In all these results we suppose that $R$ is starlike with respect to 0 and satisfies condition (b) (see (1.3)), and that $\mathcal{F}$ satisfies condition (a) (see (1.2)). In addition we suppose that $\mathcal{F}$ and $R$ meet one of the following three conditions:

(1) $\mathcal{F}$ is locally stable with respect to the family $T$ on $\bar{R}$.

(2) $\Gamma:=\bar{R} \backslash R$ is not periodic, and $\mathcal{F}$ is locally stable with respect to the family $T_{1}$ on $\bar{R}$.

(3) $\mathcal{F}$ has no multiple zeros on $\Gamma$, and is locally stable with respect to the family $T_{2}$ on $\bar{R}$.

Under these hypotheses the following holds.

Theorem 1.5. The graph of zeros $\mathcal{G}_{\mathcal{F}, R}(V)$ is of A-type.

Remark 1.6. Suppose that $\mathcal{F}$ is an arbitrary family satisfying (1.2) and that $\Gamma=$ $\bar{R} \backslash R$ is a non-periodic closed non-singular analytic curve. Let us show that a small perturbation of $\mathcal{F}$ gives a new family $\widetilde{\mathcal{F}}$ satisfying the conditions of Theorem 1.5 i.e., these conditions are generic.

To this end choose $a>0$ such that $(1+a) z \in D$ for any $z \in \bar{R}$, and $b>0$, and set

$$
\begin{gathered}
\Delta:=\left\{\left(\lambda_{n+1}, \lambda_{n+2}\right) \in \mathbb{C}^{2}:\left|\lambda_{n+1}\right|<a,\left|\lambda_{n+2}\right|<b\right\} ; \\
\widetilde{U}:=U \times \Delta ; \quad \widetilde{V}:=V \times \Delta \subset \widetilde{U} .
\end{gathered}
$$

Now introduce a new family $\widetilde{\mathcal{F}}=\left\{f_{\widetilde{\lambda}} \in \mathcal{O}(\widetilde{D}): \widetilde{\lambda} \in \widetilde{U}\right\}$, where $\widetilde{D}$ is a suitable open neigbourhood of $\bar{R}$ and

$$
f_{\widetilde{\lambda}}(z):=f_{\lambda}\left(\left(1+\lambda_{n+1}\right) z\right)+\lambda_{n+2} .
$$

It is readily seen that $\widetilde{\mathcal{F}}$ satisfies the hypotheses of Theorem 1.5 . 
Let us now formulate the second main result. In this case we preserve all the hypotheses of Theorem 1.5 except for hypotheses (1),(2),(3). The latter will be replaced by the following two conditions:

(4) $\mathcal{F}$ is locally stable with respect to the family $T_{3}$ on $\bar{R}$.

(5) For any $\lambda \in V$ there is a vector $v=v(\lambda) \in \mathbb{C}^{n}$ such that $\left|D_{v} f_{\lambda}(0)\right| \geq \delta$, and

$$
\left|D_{v} f_{\lambda}(z)-D_{v} f_{\lambda}(0)\right| \leq M \cdot|z| \quad \text { if } \quad z \in \mathbb{D}_{\rho} .
$$

Here

$$
D_{v} f_{\lambda}(z):=\lim _{t \rightarrow 0} \frac{f_{\lambda+t v}(z)-f_{\lambda}(z)}{t}, \quad t \in \mathbb{C},
$$

and $\delta>0, M>0$, and $\rho>0$ all depend only on $V$ and $\mathcal{F}$.

Under these assumptions the following holds.

Theorem 1.7. Suppose that $\Gamma=\bar{R} \backslash R$ is non-periodic and is contained in $\mathbb{D}_{r}$ with $r:=\min \left\{\rho, \frac{\delta}{2 M}\right\}$. Then the graph $\mathcal{G}_{\mathcal{F}, R}(V)$ is of A-type.

1.2. In this section we formulate some applications of our main results, illustrated by examples.

Let $f$ and $f_{1}, \ldots, f_{k}$ be non-constant entire functions on $\mathbb{C}^{k}$ and $\mathbb{C}$, respectively. Introduce a family $\mathcal{F}$ by the formula

$$
f_{\lambda}(z):=f\left(f_{1}\left(p_{1}(z)\right), \ldots, f_{k}\left(p_{k}(z)\right)\right),
$$

where $p_{j}$ is a fixed member of the space of holomorphic polynomials of degrees at most $d_{j}, 1 \leq j \leq k$, respectively, and $\lambda \in \mathbb{C}^{d}, d:=\sum_{j=1}^{k}\left(d_{j}+1\right)$, is the vector consisting of the coefficients of all the $p_{j}$.

Suppose that

(A) the complex analytic set

$$
Z:=\left\{\left(v_{1}, \ldots, v_{k}\right) \in \mathbb{C}^{d}: f\left(f_{1}\left(v_{1}\right), \ldots, f_{k}\left(v_{k}\right)\right)=0\right\}
$$

is irreducible, and

(B) there exists $\lambda_{0} \in \mathbb{C}^{d}$ such that $f_{\lambda_{0}}$ has a simple zero in $\mathbb{C} \backslash\{0\}$.

Under these hypotheses the following is true.

Corollary 1.8. For any domain $V \subset \subset \mathbb{C}^{d}$ and $R$ starlike with respect to 0 satisfying (1.3) the graph $\mathcal{G}_{\mathcal{F}, R}(V)$ is of A-type. Moreover, there exists a subset $X_{R} \subset \mathbb{C}^{d}$ of Lebesgue measure 0 such that any function $f_{\lambda} \in \mathcal{F}$ with $\lambda \in \mathbb{C}^{d} \backslash X_{R}$ admits only simple zeros on any curve $t \Gamma, t>0$.

Example 1.9. (1) Let

$$
\mathcal{F}:=\left\{f_{\lambda}(z):=\sum_{j=0}^{n} \lambda_{j} z^{j}: \lambda=\left(\lambda_{0}, \ldots, \lambda_{n}\right) \in \mathbb{C}^{n+1}\right\}
$$

be the space of holomorphic polynomials of degree at most $n$. Then Theorem 1.5 implies that for any $V \subset \mathbb{C}^{n+1}$ and any $R$ starlike with respect to 0 satisfying (1.3) the graph $\mathcal{G}_{\mathcal{F}, R}(V)$ is of $A$-type.

In particular, for $V=\mathbb{C}^{n+1}$ the set of zeros of $\mathcal{F}$ in $\bar{R}$ is naturally parameterized by points of the complex projective space $\mathbb{C P}^{n}$. In this case the corollary states that $\mathbb{C P}^{n}$ is a disjoint union of connected subanalytic subsets $V_{i}, 0 \leq i \leq n$, such that for each point from $V_{i}$ the corresponding polynomial has exactly $i$ zeros in $\bar{R}$. Moreover, any pair $i, j$ such that $\operatorname{dim}_{\mathbb{R}} \overline{V_{i}} \cap \overline{V_{j}}=2 n-1$ satisfies $|i-j|=1$. 
(2) Let $\mathcal{F}=\left\{f_{\lambda}\right\}$ be the family of generalized exponential polynomials

$$
f_{\lambda}(z)=\sum_{j=1}^{k} p_{j}(z) e^{q_{j}(z)},
$$

where $p_{j}, q_{j}$ are again holomorphic polynomials of degrees at most $p$ and $q$, respectively, and $\lambda \in \mathbb{C}^{k(p+q+2)}$ is the vector of coefficients of all the $p_{j}$ and $q_{j}$. Then for any domain $V \subset \subset \mathbb{C}^{k(p+q+2)}$ and any $R$ starlike with respect to 0 satisfying (1.3), the graph $\mathcal{G}_{\mathcal{F}, R}(V)$ is of $A$-type. In fact, the set $Z:=\left\{\left(v_{1}, \ldots, v_{2 k}\right) \in\right.$ $\left.\mathbb{C}^{2 k}: v_{1} e^{v_{2}}+\cdots+v_{2 k-1} e^{v_{2 k}}=0\right\}$ coincides with the graph of the entire function $v_{1} e^{v_{2}-v_{2 k}}+\cdots+v_{2 k-3} e^{v_{2 k-2}-v_{2 k}}$ defined on $\mathbb{C}^{2 k-1}$, and therefore is irreducible, and Corollary 1.8 can be applied.

Now we formulate an application related to dynamical systems generated by polynomial vector fields. The central problem in this area is the (still unsolved) second part of Hilbert's sixteenth problem. This problem asks whether the number of isolated closed trajectories (limit cycles) of a planar polynomial vector field is always bounded in terms of its degree (for recent developments related to Hilbert's problem see, e.g., [I). In this paper we consider a local version of this problem for complex vector fields close to the linear rotation

$$
\left\{\begin{array}{l}
\dot{x}=-y+F(x, y) \\
\dot{y}=x+G(x, y)
\end{array}\right.
$$

where the functions $F$ and $G$ are given by

$$
F(x, y)=\sum_{1 \leq i+k \leq d} a_{k i} x^{k} y^{i}, \quad G(x, y)=\sum_{1 \leq i+k \leq d} b_{k i} x^{k} y^{i}, \quad d \geq 2 .
$$

Let $\lambda \in \mathbb{C}^{d(d+3)}$ be a vector formed by an ordered collection of the coefficients of $F$ and $G$. To introduce an analytic family related to the local behavior of the solutions of (1.4) near the origin we rewrite (1.4) in polar coordinates $x=r \cos \phi, y=r \sin \phi$. Then we have

$$
\frac{d r}{d \phi}=\frac{P}{1+Q} r
$$

where $P$ and $Q$ are given by

$$
P(r, \phi):=\frac{x F(x, y)+y G(x, y)}{r^{2}}, \quad Q(r, \phi):=\frac{x G(x, y)-y F(x, y)}{r^{2}} .
$$

Now let $r\left(\phi, \lambda, r_{0}\right)$ be the analytic solution of (1.5) with initial value $r_{0} \in \mathbb{C}$ (it exists for sufficiently small vectors of the coefficients $\lambda$ and $r_{0}$, and is holomorphic in these variables). Then, by definition, $P_{\lambda}\left(r_{0}\right):=r\left(2 \pi, \lambda, r_{0}\right)$ is the Poincaré map and $d_{\lambda}\left(r_{0}\right):=P_{\lambda}\left(r_{0}\right)-r_{0}$ the displacement map of (1.5). Now we introduce the analytic family

$$
\mathcal{D}:=\left\{d_{\lambda} \in \mathcal{O}\left(\mathbb{D}_{r}\right): \lambda \in B_{c}(0, N)\right\} .
$$

Here $B_{c}(0, N)$ is the open Euclidean ball in $\mathbb{C}^{d(d+3)}$, and $N=N(d)$ and $r=r(N)$ are sufficiently small. The role of the zeros of the family $\mathcal{D}$ is as follows.

If $d_{\lambda}, \lambda \in \mathbb{R}^{d(d+3)}$, is not identically zero, then positive zeros of equation $d_{\lambda}(x)=$ 0 correspond to limit cycles of the system (1.4) situated in a small neighbourhood of the origin. 
Theorem 1.10. Suppose that $R \in \mathbb{D}_{1 / 8}$ is starlike with respect to 0 and its boundary $\Gamma$ is a non-singular, non-periodic analytic curve. Then the graph of zeros $\mathcal{G}_{\mathcal{D}, R}\left(B_{c}(0, N)\right)$ is of A-type for $N:=\frac{1}{40 \pi \sqrt{d}}$.

\section{Proofs of Theorem 1.5 and Corollary 1.8}

2.1. First we prove some auxiliary results.

Lemma 2.1. Suppose that the number of vertices of the graph $\mathcal{G}_{\mathcal{F}, R}(V)$ is at least 2. Then $\operatorname{codim}_{\mathbb{R}} \partial Z_{i}=1$ for any vertex $i \in \mathcal{G}_{\mathcal{F}, R}(V)$.

Proof. Assume, to the contrary, that there exists an $i$ such that the real codimension of the subanalytic set $\partial Z_{i}:=\overline{Z_{i}} \backslash Z_{i}$ is greater than 1 . According to our hypothesis the open set $Y:=V \backslash \overline{Z_{i}}$ is non-empty. Thus $V \backslash \partial Z_{i}$ is the disjoint union $Z_{i} \sqcup Y$. But according to a topological property of subanalytic sets, see [Ha], the pair $\left(\partial Z_{i}, V\right)$ is triangulable. Since $\operatorname{codim}_{\mathbb{R}} \partial Z_{i} \geq 2$, this implies that $V \backslash \partial Z_{i}$ is connected. This contradiction proves the lemma.

Lemma 2.2. Suppose that $\mathcal{F}$ is locally stable with respect to the family $T_{3}$ on $\bar{R}$. Let $S \subset V \subset \mathbb{C}^{n}$ be the subanalytic set from Proposition 1.2 Then:

(1) $S \backslash C_{\mathcal{F}}$ is of pure dimension $2 n-1$; that is, for any $x \in S \backslash C_{\mathcal{F}}$ and any sufficiently small open Euclidean ball $B_{x}$ centered at $x, \operatorname{dim}_{\mathbb{R}} B_{x} \cap S=2 n-1$.

(2) $V \backslash S$ contains the sets $Z_{m}$ and $Z_{M}$, where $m$ and $M$ are infimum and supremum of $\mathcal{N}_{\mathcal{F}, R}$ on $V \backslash C_{\mathcal{F}}$.

Proof. (1) Assume, to the contrary, that there are a point $x \in S \backslash C_{\mathcal{F}}$ and a sufficiently small open Euclidean ball $B_{x}$ centered at $x$ such that the real dimension of $S_{x}:=B_{x} \cap S$ is at most $2 n-2$. Since $S_{x}$ is subanalytic, without loss of generality we may assume that $x \in S$ is a smooth point and $S_{x} \subset B_{x}$ is a smooth submanifold. Next, from the local stability of $\mathcal{F}$ it follows that there is a continuous map $\beta_{x}(t)$ : $I_{x} \rightarrow B_{x}$ of a small open interval $I_{x}:=\{t \in \mathbb{R}:|t-1|<r(x)\}$ such that

$$
f_{\beta_{x}(t)}(z)=f_{x}(t z), \quad z \in \bar{R}
$$

Since $x \notin C_{\mathcal{F}}$, the set $K:=\left\{t \in I_{x}: \beta_{x}(t) \in S\right\}$ is finite (otherwise, from the definitions of $\beta_{x}$ and $S$ we obtain that $f_{x}$ is identically zero). Thus, diminishing $I_{x}$ if necessary, we may assume that $K=\{1\}$. Now from the definitions of $\beta_{x}$ and $S$ it follows that the number $\mathcal{N}_{\mathcal{F}, R}\left(\beta_{x}(1+\epsilon)\right)-\mathcal{N}_{\mathcal{F}, R}\left(\beta_{x}(1-\epsilon)\right)$ for any positive $\epsilon<r(x)$ is positive and equals the number of zeros of $f_{x}$ on $\Gamma$. But according to our assumption, $B_{x} \backslash S_{x}$ is connected. In particular, there is a continuous path joining $\beta_{x}(1+\epsilon)$ and $\beta_{x}(1-\epsilon)$ inside $B_{x} \backslash S$. Then from the formula

$$
\mathcal{N}_{\mathcal{F}, R}(\lambda):=\frac{1}{2 \pi i} \int_{\Gamma} \frac{f_{\lambda}^{\prime}(z)}{f_{\lambda}(z)} d z
$$

valid for $\lambda \notin S$, it follows that

$$
\mathcal{N}_{\mathcal{F}, R}\left(\beta_{x}(1+\epsilon)\right)=\mathcal{N}_{\mathcal{F}, R}\left(\beta_{x}(1-\epsilon)\right) .
$$

This contradiction proves (1).

(2) Since $\mathcal{N}_{\mathcal{F}, R}$ is upper semicontinuous on $V$, it attains the value $m$ on a relatively open subset of $V$. This implies that $Z_{m} \neq \emptyset$ (because $\left.\operatorname{dim}_{\mathbb{R}} S=2 n-1\right)$. Next, assume, to the contrary, that $Z_{M}=\emptyset$. Since $\mathcal{N}_{\mathcal{F}, R}$ is integer-valued and $M<\infty$, this assumption implies that there exists a smooth point $x \in S \backslash C_{\mathcal{F}}$ such that $\mathcal{N}_{\mathcal{F}, R}(x)=M$. Let us consider the map $\beta_{x}(t): I_{x} \rightarrow V$ defined as in 
the proof of part (1). From its definition it follows that there is an $i \in \mathbb{Z}_{+}$such that $\beta_{x}(1+\epsilon) \in Z_{i}$ for any positive $\epsilon<r(x)$. This implies that $i \geq M$. Hence $Z_{i}=Z_{M} \neq \emptyset$. This contradiction proves the lemma.

2.2. Proof of Theorem 1.5. Consider a subset $Z_{i} \subset V \backslash S$. According to Lemma 2.1 there is an open Euclidean ball $B$ centered at a point from $\partial Z_{i}$ such that $B \cap S \subset \partial Z_{i}$ is a smooth closed submanifold of $B$ of real codimension 1, and $B \backslash S=U_{1} \sqcup U_{2}$, where $U_{1}$ and $U_{2}$ are open and connected, and, say, $U_{1} \subset Z_{i}$, $U_{2} \subset Z_{j}$ for some $j$ (not necessarily distinct from $i$ ). In particular, $Y_{i j}:=B \cap S$ is the common boundary of $Z_{i}$ and $Z_{j}$, and $B \backslash Y_{i j}=\left(Z_{i} \cap B\right) \sqcup\left(Z_{j} \cap B\right)$.

Choose a point $y \in Y_{i j}$, and let $z_{1}, \ldots, z_{k}$ be the set of distinct zeros of $f_{y}$ on $\Gamma$ of orders $\mu_{1}, \ldots, \mu_{k}$, respectively.

Lemma 2.3. Under the hypotheses of Theorem 1.5 we have

$$
|i-j|=\sum_{s=1}^{k} \mu_{s}>0 .
$$

Proof. From the local stability of $\mathcal{F}$ with respect to the family $T_{3}$ on $\bar{R}$ it follows that there exists a continuous map $\beta_{y}: I_{y} \rightarrow B \subset V$ of a small open interval $I_{y}:=\{t \in \mathbb{R}:|t-1|<r(y)\}$ such that

$$
f_{\beta_{y}(t)}(z)=f_{y}(t z), \quad z \in \bar{R} .
$$

Then, as in the proof of Lemma 2.2 the set $K:=\left\{t \in I_{y}: \beta_{y}(t) \in S\right\}$ coincides with $\{1\}$ for a sufficiently small $r(y)$. Let us show that for such $r(y)$ the image $\beta_{y}\left(I_{y}\right)$ does not belong entirely to either $\overline{Z_{i}}$ or $\overline{Z_{j}}$. In fact, assuming the opposite we obtain for any $t_{1}, t_{2} \in I_{y}, t_{1}<1<t_{2}$, that

$$
\mathcal{N}_{\mathcal{F}, R}\left(\beta_{y}\left(t_{1}\right)\right)=\mathcal{N}_{\mathcal{F}, R}\left(\beta_{y}\left(t_{2}\right)\right) .
$$

Here, by definition, the value on the left is the number of zeros of $f_{y}$ in $t_{1} \bar{R}$ and the value on the right is the number of zeros of $f_{y}$ in $t_{2} \bar{R}$. But $t_{1} \bar{R} \subset \bar{R} \subset t_{2} \bar{R}$ (since $R$ is starlike with respect to 0 ) and $f_{y}$ has zeros on the boundary $\Gamma$ of $\bar{R}$. Thus we get

$$
\mathcal{N}_{\mathcal{F}, R}\left(\beta_{y}\left(t_{1}\right)\right)<\mathcal{N}_{\mathcal{F}, R}\left(\beta_{y}\left(t_{2}\right)\right) .
$$

This contradiction shows that we have, say, $\beta_{y}\left(I_{y}^{\prime}\right) \subset Z_{j}$ and $\beta_{y}\left(I_{y}^{\prime \prime}\right) \subset Z_{i}$, for $I_{y}^{\prime}=\{1<t<1+r(y)\}$ and $I_{y}^{\prime \prime}=\{1-r(y)<t<1\}$, and

$$
j-i=\sum_{s=1}^{k} \mu_{s}>0 .
$$

Next, we will show that the set of zeros of $f_{y}$ on $\Gamma$ consists of a single point, i.e., $k=1$. In the proof we will use the following result.

Lemma 2.4. Let $z_{1}, \ldots, z_{k} \in \Gamma$ be pairwise distinct points.

(1) There is a nowhere dense subset $L \subset \mathbb{C}$ such that for any $w \in \mathbb{C} \backslash L$ there are pairwise distinct $r_{1}, \ldots, r_{k} \in \mathbb{R}_{+}$so that $z_{i}-w \in r_{i} \Gamma, 1 \leq i \leq k$.

(2) If $\Gamma$ is non-periodic, then there is a finite subset $M \subset S^{1}$ such that for any $w \in S^{1} \backslash M$ there are pairwise distinct $r_{1}, \ldots, r_{k} \in \mathbb{R}_{+}$so that $w \cdot z_{i} \in r_{i} \Gamma$, $1 \leq i \leq k$. 
Proof. (1) Each point $z \in \mathbb{C} \backslash\{0\}$ can be written uniquely as $z=r \cdot \gamma, r \in \mathbb{R}_{+}, \gamma \in \Gamma$. Since $\Gamma$ is a non-singular analytic curve, the transition from Cartesian coordinates on $\mathbb{R}^{2} \backslash\{0\}$ to the coordinates $(r, \gamma)$ is analytic. For each $i$ consider the map $\mathbb{C} \rightarrow \mathbb{C}$, $w \mapsto z_{i}-w$. For $w \neq z_{i}$ it can be written as $g_{i}(w) \cdot h_{i}(w), g_{i}(w) \in \mathbb{R}_{+}, h_{i}(w) \in \Gamma$, where $g_{i}, h_{i}$ are analytic on $\mathbb{C} \backslash\left\{z_{i}\right\}$ and $g_{i}$ can be extended continuously at $z_{i}$. The identity $g_{i}(w)=g_{j}(w)$ for $i \neq j$ means that there is an $r \in \mathbb{R}_{+}$such that $z_{i}-w$ and $z_{j}-w$ belong to $r \Gamma$. Let

$$
L:=\left\{w \in \mathbb{C}: g_{i}(w)=g_{j}(w) \text { for some } i \neq j\right\} .
$$

Then each $w \notin L$ satisfies the required statement. Let us show that $L \subset \mathbb{C}$ is nowhere dense. Notice that by the definition $L \backslash\left\{z_{1}, \ldots, z_{k}\right\}$ is an analytic subset of $\mathbb{C} \backslash\left\{z_{1}, \ldots, z_{k}\right\}$. Thus it suffices to check that each $L_{i j}:=\left\{w \in \mathbb{C}: g_{i}(w)=g_{j}(w)\right\}$, $i \neq j$, is a proper subset of $\mathbb{C}$. In fact, since $z_{i} \neq z_{j}$ we have $0=g_{i}\left(z_{i}\right) \neq g_{j}\left(z_{i}\right)$ and $0=g_{j}\left(z_{j}\right) \neq g_{i}\left(z_{j}\right)$, implying that $z_{i}, z_{j} \notin L_{i j}$. This completes the proof of (1).

(2) In polar coordinates $(r, \theta) \in \mathbb{R}_{+} \times \mathbb{R}$ the curve $\Gamma$ is the graph of a positive $2 \pi$-periodic analytic function $r=f(\theta)$. The map $z \mapsto w \cdot z, w=e^{i \phi} \in S^{1}$, in these coordinates is defined by the translation $(r, \theta) \mapsto(r, \theta+\phi)$. Suppose that $z_{i}=\left(f\left(\theta_{i}\right), \theta_{i}\right)$ for some $\theta_{i} \in[0,2 \pi), 1 \leq i \leq k$. Assume, to the contrary, that there are $i \neq j$, an infinite sequence $\left\{\phi_{k}\right\} \subset[0,2 \pi)$, and sequences $\left\{r_{k}\right\} \subset \mathbb{R}_{+}$, $\left\{y_{k i}\right\},\left\{y_{k j}\right\} \subset[0,4 \pi)$ such that for any $k$,

$$
\left(f\left(\theta_{i}\right), \theta_{i}+\phi_{k}\right)=\left(r_{k} \cdot f\left(y_{k i}\right), y_{k i}\right) \text { and }\left(f\left(\theta_{j}\right), \theta_{j}+\phi_{k}\right)=\left(r_{k} \cdot f\left(y_{k j}\right), y_{k j}\right) .
$$

This implies that for any $k$,

$$
\frac{f\left(y_{k i}\right)}{f\left(y_{k i}-\left(\theta_{i}-\theta_{j}\right)\right)}=\frac{f\left(\theta_{i}\right)}{f\left(\theta_{j}\right)} .
$$

But by definition $\left\{y_{k i}\right\}$ has a limit point in $[0,4 \pi]$, and $f$ is $2 \pi$-periodic and analytic. Thus we have

$$
\frac{f(t)}{f\left(t-\left(\theta_{i}-\theta_{j}\right)\right)}=\frac{f\left(\theta_{i}\right)}{f\left(\theta_{j}\right)}, \quad t \in \mathbb{R} .
$$

We will prove now that $f\left(\theta_{i}\right)=f\left(\theta_{j}\right)$. Suppose first that $f\left(\theta_{i}\right)>f\left(\theta_{j}\right)$. Let $t_{\text {max }} \in[0,2 \pi)$ be such that $f\left(t_{\max }\right)=\max _{\mathbb{R}} f$. Then from the above identity we obtain the contradiction

$$
1 \geq \frac{f\left(t_{\max }+\left(\theta_{i}-\theta_{j}\right)\right)}{f\left(t_{\max }\right)}=\frac{f\left(\theta_{i}\right)}{f\left(\theta_{j}\right)}>1 .
$$

Thus $f\left(\theta_{i}\right) \leq f\left(\theta_{j}\right)$. Similarly one can show that $f\left(\theta_{i}\right) \geq f\left(\theta_{j}\right)$, which gives the required equality. This means that $f(t)$ is periodic with the period $\left|\theta_{i}-\theta_{j}\right| \in(0,2 \pi)$. Then it is readily seen that $\left|\theta_{i}-\theta_{j}\right|=2 \pi q$ for some $q \in \mathbb{Q}$, and so $f(t)$ is periodic with a period $\frac{2 \pi}{l}, l \in \mathbb{N}, l>1$. This contradicts the non-periodicity of $\Gamma$.

The proof of the lemma is complete.

Lemma 2.5. Let $y \in Y_{i j}$ be as in Lemma 2.3. Then the set of zeros of $f_{y}$ on $\Gamma$ consists of a single point.

Proof. In order to prove the lemma it suffices to obtain the required statement for $\mathcal{F}$ locally stable with respect to the families $T_{2}$ and $T_{3}$ on $\bar{R}$. In what follows we retain the notation of Lemma 2.3 . 
(A) Suppose that $\mathcal{F}$ is locally stable with respect to the family $T_{2}$ on $\bar{R}$. Diminishing, if necessary, $r(y)$ in Lemma 2.3, we obtain by definition that there is a continuous map $\widetilde{\beta}_{y}: I_{y} \times \mathbb{D}_{r(y)} \rightarrow B$ such that

$$
f_{\tilde{\beta}_{y}(t, w)}(z)=f_{y}(t z+w), \quad z \in \bar{R} .
$$

Here $\widetilde{\beta}_{y}(t, 0)=\beta_{y}(t)$. Using the definition of $\beta_{y}$, from Lemma 2.3 we obtain that for $s:=\frac{r(y)}{2}$ there is a positive $s^{\prime} \leq s$ such that for any $w \in \mathbb{D}_{s^{\prime}}$ we have

$$
\widetilde{\beta}_{y}(1-s, w) \in Z_{i}, \quad \text { and } \quad \widetilde{\beta}_{y}(1+s, w) \in Z_{j} .
$$

Let $I_{s}:=\{t \in \mathbb{R}:|t-1|<s\}, \beta_{y, w}(t):=\widetilde{\beta}_{y}(t, w)$, and $K_{w}:=\beta_{y, w}\left(I_{s}\right) \cap Y_{i j}(\neq \emptyset)$. We check that for any $w \in \mathbb{D}_{s^{\prime}}$

$$
\#\left\{t \in I_{s}: \beta_{y, w}(t) \in K_{w}\right\} \leq k .
$$

In fact, $\beta_{y, w}(t) \in Y_{i j}$ for some $t \in I_{s}$ means that there is a $z^{\prime} \in \Gamma$ such that $f_{y}\left(t z^{\prime}+w\right)=0$. But then, assuming that $s$ is sufficiently small, we obtain that $t z^{\prime}+w$ coincides with some $z_{i} \in \Gamma$. Since $z^{\prime}=\frac{z_{i}-w}{t}$ and $\Gamma$ is non-singular analytic and starlike with respect to 0 , for any $i, 1 \leq i \leq k$, there is at most one $t \in I_{y}$ such that $\frac{z_{i}-w}{t} \in \Gamma$. This implies (2.1). Hence there exist a subinterval $J:=[a, b] \subset I_{s}$ and a point $t \in J$ such that

$$
\widetilde{\beta}_{y}(t, w) \in Y_{i j}, \quad \widetilde{\beta}_{y}((t, b], w) \subset Z_{j}, \quad \widetilde{\beta}_{y}([a, t), w) \subset Z_{i} .
$$

In the rest of the proof of part (A) we assume that $w \in \mathbb{D}_{s^{\prime}} \backslash L$ with $L$ defined as in Lemma 2.4 (1). Further, by the definition of $J$ and $\widetilde{\beta}_{y}$, for any $t_{1} \in[a, t)$ and $t_{2} \in(t, b]$ we have

$$
i=\mathcal{N}_{\mathcal{F}, R}\left(\widetilde{\beta}_{y}\left(t_{1}, w\right)\right)<\mathcal{N}_{\mathcal{F}, R}\left(\widetilde{\beta}_{y}\left(t_{2}, w\right)\right)=j .
$$

Since $\mathcal{N}_{\mathcal{F}, R}$ is an upper semicontinuous function, this implies that

$$
\mathcal{N}_{\mathcal{F}, R}\left(\widetilde{\beta}_{y}(t, w)\right)=j .
$$

Note that $t \Gamma+w$ is the boundary of the domain $\bigcup_{q<t}(q R+w)$. Thus from the above identities and from the definition of $\widetilde{\beta}_{y}$ we obtain that $j$ equals $i$ plus the number of the zeros of $f_{y}$ on $t \Gamma+w$. But, since $s$ is sufficiently small, each zero of $f_{y}$ on $t \Gamma+w$ coincides with one of the $z_{l}, 1 \leq l \leq k$. Since $w \notin L$, there is only one such $l$. In particular,

$$
j=i+\mu_{l} \quad \text { and } \quad \mu_{r}=0 \quad \text { if } \quad r \neq l .
$$

So, in this case, the set of zeros of $f_{y}$ on $\Gamma$ consists of a single point.

(B) Suppose now that $\mathcal{F}$ is locally stable with respect to the family $T_{3}$ on $\bar{R}$. Then without loss of generality we may assume that $r(y)$ in Lemma 2.3 is so small that there is a continuous map $\beta_{y}: D(1 ; r(y)) \rightarrow B, D(1 ; r(y)):=1+\mathbb{D}_{r(y)}$, given by the formula

$$
f_{\beta(w)}(z)=f_{y}(w z), \quad z \in \bar{R} .
$$

(Here $\left.\beta_{y}\right|_{I_{y}}$ is the same as in Lemma 2.3.) Next, from the properties of $\left.\beta_{y}\right|_{I_{y}}$ proved in Lemma 2.3 it follows that for $s=\frac{r(y)}{2}$ we can find a positive $s^{\prime} \leq s$ such that for any $w \in S^{1} \cap D\left(1, s^{\prime}\right)$ we have

$$
\beta_{y}(w(1-s)) \in Z_{i}, \quad \text { and } \quad \beta_{y}(w(1+s)) \in Z_{j} .
$$


Then applying arguments similar to those used in part (A) of the proof, for each $w \in S^{1} \cap D\left(1, s^{\prime}\right)$ we can find a subinterval $J:=[a, b] \subset I_{s}$ and a point $t \in J$ such that

$$
\beta_{y}(w t) \in Y_{i j}, \quad \beta_{y}((w t, w b]) \subset Z_{j}, \quad \beta_{y}([w a, w t)) \subset Z_{i} .
$$

Moreover, as before, we obtain that $j$ equals $i$ plus the number of the zeros of $f_{y}$ on $w t \Gamma$. Then for a sufficiently small $r(y)$ the latter implies that each zero of $f_{y}$ on $w t \Gamma$ coincides with some $z_{l}, 1 \leq l \leq k$. Now choose $w$ such that $w^{-1} \notin M$ with $M$ defined as in Lemma 2.4 (2). Then for such $w$ this lemma implies that the set of zeros of $\left.f_{y}\right|_{w t \Gamma}$ consists of a single point. Then, as above, we deduce that the set of zeros of $f_{y}$ on $\Gamma$ consists of a single point, as well.

The proof of the lemma is complete.

Finally we prove

Lemma 2.6. Under the hypotheses of Theorem 1.5 the zero of $f_{y}$ on $\Gamma$ has order 1.

Proof. To prove the lemma we will point out a point $y^{\prime} \in Y_{i j}$ such that all zeros of $\left.f_{y^{\prime}}\right|_{\Gamma}$ are of order 1 . Then according to Lemma 2.5 we will get that the set of zeros of $f_{y^{\prime}}$ on $\Gamma$ is a single point. Finally, applying the identity of Lemma 2.3 we will obtain that $|i-j|=1$. This will complete the proof of the lemma, and together with the statement of Lemma 2.2 (2) will complete the proof of the theorem.

First we will find such a $y^{\prime} \in Y_{i j}$ under hypothesis (3) of the theorem. Let

$$
S_{d}:=\left\{(w, \lambda) \in \Gamma \times V: f_{\lambda}(w)=\frac{d f_{\lambda}}{d z}(w)=0\right\} .
$$

By $p_{1}: \Gamma \times V \rightarrow \Gamma$ and $p_{2}: \Gamma \times V \rightarrow V$ we denote the natural projections. Since $\mathcal{F}$ has no multiple zeros on $\Gamma$, we have $\operatorname{dim}_{\mathbb{R}} p_{1}^{-1}(w) \leq 2 n-4\left(\right.$ where $\operatorname{dim}_{\mathbb{R}} V=2 n$ ). Therefore $\operatorname{dim}_{\mathbb{R}} S_{d} \leq 2 n-3$, which implies that $\operatorname{dim}_{\mathbb{R}} p_{2}\left(S_{d}\right) \leq 2 n-3$. Since $\operatorname{dim}_{\mathbb{R}} Y_{i j}=2 n-1$, the latter inequality shows that there is a point $y^{\prime} \in Y_{i j} \backslash p_{2}\left(S_{d}\right)$. By the definition, all zeros of $\left.f_{y^{\prime}}\right|_{\Gamma}$ are of order 1 .

Now, let us prove the lemma under hypotheses (1) and (2).

Let $z_{1}$ be the zero of $f_{y}$ on $\Gamma$ of order $\mu$ (here $y$ is the same as in Lemma 2.3). Then in a small open neighbourhood $O$ of $\Gamma$ we have

$$
f_{y}(z)=g(z) \cdot\left(z-z_{1}\right)^{\mu}
$$

where $\left.g\right|_{O}$ is holomorphic and nowhere 0 . Let us consider the equation

$$
g(z)\left(z-z_{1}\right)^{\mu}=-c .
$$

According to the Rouché theorem there are an open neighbourhood $\widetilde{O} \subset O$ of $\Gamma$ and a $\delta>0$ such that for any $c \in \mathbb{D}_{\delta}$ equation (2.2) has $\mu$ zeros in $\widetilde{O}$. Doing the change of variables $w:=(g(z))^{1 / \mu}\left(z-z_{1}\right)$, valid in a small open neighbourhood $O_{1} \subset \widetilde{O}$ of $z_{1}$, we obtain $w^{m}=-c$. From here, diminishing $\delta$ if necessary, we conclude that for any $c \in \mathbb{D}_{\delta}, c \neq 0$, equation (2.2) has $\mu$ distinct zeros in $O_{1}$.

Next, since $\mathcal{F}$ is locally stable with respect to the families $T$ and $T_{1}$ on $\bar{R}$, we can find a small positive $\epsilon \leq \delta$ such that

(a) $t z \in \widetilde{O}$ for any $t \in I_{\epsilon}:=\{t \in \mathbb{R}:|t-1|<\epsilon\}, z \in \Gamma$, and

(b) there is a continuous map $\beta_{y}^{\prime}: I_{\epsilon} \times \mathbb{D}_{\epsilon} \rightarrow B$ given by

$$
f_{\beta_{y}^{\prime}(t, c)}(z)=f_{y}(t z)+c, \quad z \in \bar{R} \text {. }
$$


Now, as in the proof of Lemma 2.5, we can find a positive $\epsilon^{\prime} \leq \epsilon$ such that for each $c \in \mathbb{D}_{\epsilon^{\prime}}$,

$$
\beta_{y}^{\prime}(1-\epsilon, c) \in Z_{i} \quad \text { and } \quad \beta_{y}^{\prime}(1+\epsilon, c) \in Z_{j} .
$$

(Here we retain the assumptions of Lemma 2.3.) Suppose that $c \in \mathbb{D}_{\epsilon^{\prime}} \backslash\{0\}$. Since the intersection $\beta_{y}^{\prime}\left(I_{\epsilon}, c\right) \cap Y_{i j} \neq \emptyset$, there is a point $s \in I_{\epsilon}$ such that $f_{\beta_{y}^{\prime}(s, c)}(z):=$ $f_{y}(s z)+c$ has a zero on $\Gamma$. But from our definitions of $\epsilon$ and $\delta$ it follows that $f_{\beta_{y}^{\prime}(s, c)}$ has at most $\mu$ distinct zeros on $\Gamma$, each of order 1 . Therefore the point $y^{\prime}:=\beta_{y}^{\prime}(s, c) \in Y_{i j}$ satisfies the property described at the beginning of the proof of the lemma. This implies that $|i-j|=1$.

The proof of the theorem is complete.

2.3. Proof of Corollary 1.8, Clearly, the family $\mathcal{F}$ satisfies condition (1.2) (for otherwise, the function $f\left(f_{1}\left(z_{1}\right), \ldots, f_{k}\left(z_{k}\right)\right)$ is identically zero). First we will show that $\mathcal{F}$ has no multiple zeros on $\Gamma$.

For any $w \in \Gamma$ we set

$$
Z_{w}:=\left\{\lambda \in \mathbb{C}^{d}: f_{\lambda}(w)=f_{\lambda}^{\prime}(w)=0\right\} .
$$

Assume, to the contrary, that there exists a $w_{0} \in \Gamma$ such that $Z_{w_{0}}$ has complex codimension 1. Then $Z_{w}$ has complex codimension 1 for any $w \neq 0$. This follows from the identities $f_{\lambda}\left(w_{0}\right)=f_{\widetilde{\lambda}}(w)$ and $f_{\lambda}^{\prime}\left(w_{0}\right)=\frac{w}{w_{0}}\left(f_{\tilde{\lambda}}^{\prime}(w)\right)$, where

$$
f_{\tilde{\lambda}}(z)=f\left(f_{1}\left(\widetilde{p}_{1}(z)\right), \ldots, f_{k}\left(\widetilde{p}_{k}(z)\right)\right), \quad \widetilde{p}_{j}(z)=p_{j}\left(w_{0} z / w\right) .
$$

Thus it suffices to consider $w_{0}=1$. Observe that the analytic hypersurface

$$
X_{1}=\left\{\lambda \in \mathbb{C}^{d}: f\left(f_{1}\left(p_{1}(1)\right), \ldots, f_{k}\left(p_{k}(1)\right)\right)=0\right\} \subset \mathbb{C}^{d}
$$

is irreducible because it is isomorphic to $Z \times \mathbb{C}^{d-k}$, where $Z$ is irreducible by hypothesis (A) of the corollary. Since according to our assumption $Z_{1} \subset \mathbb{C}^{d}$ is a closed analytic subset of complex codimension 1 and $Z_{1} \subset X_{1}$, from irreducibility of $X_{1}$ we obtain that $Z_{1}=X_{1}$. Hence, $Z_{w}=X_{w}:=\left\{\lambda \in \mathbb{C}^{d}: f_{\lambda}(w)=0\right\}$ for any $w \neq 0$. This means that any non-zero root of each $f_{\lambda}$ is of order $>1$, which contradicts hypothesis (B) of the corollary. Therefore we conclude that the family $\mathcal{F}$ has no multiple zeros on $\Gamma$. Also, by definition, $\mathcal{F}$ is locally stable with respect to the family $T_{2}$ on $\bar{R}$. Thus Theorem 1.5 implies that $\mathcal{G}_{\mathcal{F}, R}(V)$ is of A-type for any domain $V \subset \subset \mathbb{C}^{d}$ and any $R \subset \subset \mathbb{C}$ starlike with respect to 0 satisfying (1.3).

Now we prove the second statement of the corollary.

By definition, for each $\lambda \in\left(\mathbb{C}^{d}\right)^{*}:=\mathbb{C}^{d} \backslash\{0\}$ and each $t \in \mathbb{R}_{+}$we have $f_{\lambda}(t z):=$ $f_{h(t, \lambda)}(z)$, where

$$
\begin{gathered}
f_{\lambda}(z)=f\left(f_{1}\left(p_{1}(z)\right), \ldots, f_{k}\left(p_{k}(z)\right)\right), \\
f_{h(t, \lambda)}(z):=f\left(f_{1}\left(p_{t, 1}(z)\right), \ldots, f_{k}\left(p_{t, k}(z)\right)\right), \quad p_{t, j}(z):=p_{j}(t z) .
\end{gathered}
$$

Let $L_{\lambda} \subset\left(\mathbb{C}^{d}\right)^{*}$ be the image of $\mathbb{R}_{+}$under the map $h(\cdot, \lambda): \mathbb{R}_{+} \rightarrow\left(\mathbb{C}^{d}\right)^{*}$. It is readily seen that the required result follows from the first part of the corollary and from the following statement:

$L_{\lambda}$ intersects the set $S:=\left\{v \in \mathbb{C}^{d}: f_{v}(z)=0 \quad\right.$ for some $\left.z \in \Gamma\right\}$ in smooth points for almost all $\lambda \in\left(\mathbb{C}^{d}\right)^{*}$. 
In the rest of the proof we will show that the above statement is true.

Let $\mathbb{C}^{d_{j}+1}$ be the space of vectors $v_{j}=\left(v_{j 0}, \ldots, v_{j d_{j}}\right)$ composed from coefficients of the polynomial $p_{j}(z):=\sum_{0 \leq k \leq d_{j}} v_{j k} z^{k}$ in the definition of $f_{\lambda}$. The action of $\mathbb{R}_{+}$ on $\left(\mathbb{C}^{d}\right)^{*}$ determined by $h$ induces a similar action $h_{j}$ of $\mathbb{R}_{+}$on $\mathbb{C}^{d_{j}+1}$,

$$
h_{j}\left(t, v_{j}\right)=\left(v_{j 0}, t v_{j 1}, \ldots, t^{d_{j}} v_{j d_{j}}\right), \quad v_{j}=\left(v_{j 0}, \ldots, v_{j d_{j}}\right),
$$

so that

$$
h(t, v):=\left(h_{1}\left(t, v_{1}\right), \ldots, h_{k}\left(t, v_{k}\right)\right), \quad v=\left(v_{1}, \ldots, v_{k}\right) \in\left(\mathbb{C}^{d}\right)^{*}, \quad t \in \mathbb{R}_{+} .
$$

Set

$$
V:=\left\{v \in \mathbb{C}^{d}: \sum_{1 \leq j \leq k}\left(\sum_{1 \leq s \leq d_{j}}\left|v_{j s}\right|^{\frac{2 d !}{s}}\right)=1\right\} .
$$

Then it is easy to see that $\left.h\right|_{\mathbb{R}_{+} \times V}: \mathbb{R}_{+} \times V \rightarrow\left(\mathbb{C}^{d}\right)^{*}$ is an analytic isomorphism. By $\widetilde{\pi}:\left(\mathbb{C}^{d}\right)^{*} \rightarrow V$ we denote the composite of the projection $\pi: \mathbb{R}_{+} \times V \rightarrow V$ and $\left(\left.h\right|_{\mathbb{R}_{+} \times V}\right)^{-1}$. Next we set

$$
B_{i}:=\left\{v \in \mathbb{C}^{d}: \frac{1}{i} \leq \sum_{1 \leq j \leq k} \sum_{1 \leq s \leq d_{j}}\left|v_{j s}\right|^{\frac{2 d !}{s}} \leq i\right\}, \quad S_{i}:=S \cap B_{i}, \quad i=1,2, \ldots
$$

Then each $S_{i}$ is subanalytic and $\widetilde{\pi}: S_{i} \rightarrow V$ is a proper map. In particular, $\widetilde{\pi}\left(S_{i}\right) \subset V$ is subanalytic and $\widetilde{\pi}(S)=\bigcup_{i} \widetilde{\pi}\left(S_{i}\right)$.

Let $C \subset \mathbb{C}^{d}$ be the central set of the family $\mathcal{F}$ and $C_{i}:=C \cap B_{i}$. Since, by definition, $\operatorname{codim}_{\mathbb{R}} C \geq 2$, we have $\operatorname{codim}_{\mathbb{R}} \widetilde{\pi}\left(C_{i}\right) \geq 1$.

Further, let $K \subset S_{i}$ be a compact subset. We will check that there is a constant $M_{K}<\infty$ such that for any $v \in \widetilde{\pi}\left(S_{i}\right) \backslash \widetilde{\pi}\left(C_{i}\right)$ we have

$$
\#\left\{\tilde{\pi}^{-1}(v) \cap K\right\} \leq M_{K} .
$$

Actually, $\widetilde{\pi}^{-1}(v)$ coincides with the set $\left\{h(r, v) \in\left(\mathbb{C}^{d}\right)^{*}: r>0\right\}$. The condition $h(r, v) \in S$ means that the function $f_{v}$ has zeros on $r \Gamma$. If, in addition, $h(r, v) \in S_{i}$, then $r$ lies in the interval $A_{i}:=\left\{r \in \mathbb{R}_{+}:\left(\frac{1}{i}\right)^{1 / 2 d !} \leq r \leq i^{1 / 2 d !}\right\}$. This means that $\#\left\{\tilde{\pi}^{-1}(v) \cap K\right\}$ is less than or equal to the number of zeros of $f_{v}$ in the compact domain $R_{i}$ bounded by $\left(\frac{1}{i}\right)^{1 / 2 d !} \Gamma$ and $i^{1 / 2 d !} \Gamma$. Since according to our assumption $v \notin C$, this number is finite. Now the condition $\widetilde{\pi}^{-1}(v) \in K$ means that $v$ varies in a compact subset $T \subset V$. In this case it is well known (cf. [Br2]) that the number of zeros of $f_{v}, v \in T$, in the compact $R_{i}$ is uniformly bounded by a constant $M_{K}<\infty$.

From the above result it follows that for any compact subanalytic subset $K \subset$ $S_{i} \backslash C$ we have $\operatorname{dim}_{\mathbb{R}} K=\operatorname{dim}_{\mathbb{R}} \widetilde{\pi}(K)$. Therefore $\operatorname{dim}_{\mathbb{R}} S_{i}=\operatorname{dim}_{\mathbb{R}} \widetilde{\pi}\left(S_{i}\right)$. But according to hypothesis (B) of the corollary, $S_{i} \neq \emptyset$ for some $i$. In particular, by Lemma $2.2(1), \operatorname{dim}_{\mathbb{R}} S_{i}=2 d-1$. Thus $\operatorname{dim}_{\mathbb{R}} \widetilde{\pi}\left(S_{i}\right)=\operatorname{dim}_{\mathbb{R}} V$.

Let $S_{1 i} \subset S_{i}$ be the set containing all singular points of $S_{i}$ and all points from $C_{i}$. Then $S_{1 i}$ is a subanalytic subset of $S_{i}$ of codimension $\geq 1$. In particular, $\operatorname{dim}_{\mathbb{R}} \tilde{\pi}\left(S_{1 i}\right)<\operatorname{dim}_{\mathbb{R}} \widetilde{\pi}\left(S_{i}\right)$. This means that the subanalytic set $\widetilde{\pi}\left(S_{1 i}\right)$ is of Lebesgue measure zero in $V$ but $\tilde{\pi}\left(S_{i}\right)$ has an exhaustion by subsets of positive measure. Finally we have $X_{1}:=\bigcup_{i} \widetilde{\pi}\left(S_{1 i}\right)$ is of measure 0 in $\widetilde{\pi}(S)=\bigcup_{i} \widetilde{\pi}\left(S_{i}\right)$. It remains to set $X_{R}:=\widetilde{\pi}^{-1}\left(X_{1}\right)$. Then according to our construction $X_{R}$ is of Lebesgue measure 0 in $\mathbb{C}^{d}$, and for each $\lambda \in\left(\mathbb{C}^{d}\right)^{*} \backslash X_{R}$ the set $L_{\lambda}:=h\left(\mathbb{R}_{+}, \lambda\right)$ intersects $S$ in smooth points only.

The proof of the corollary is complete. 


\section{Proofs of Theorems 1.7 And 1.10}

3.1. Proof of Theorem 1.7, We retain the notation of the proof of Theorem 1.5

Let $Y_{i j} \subset B$ be the smooth boundary of real codimension 1 of $Z_{i} \cap B$ and $Z_{j} \cap B$, where $B \subset V$ is a an open Euclidean ball. Choose a $y \in Y_{i j}$. Since, by hypothesis (4) of the theorem, $\mathcal{F}$ is locally stable with respect to the family $T_{3}$ on $\bar{R}$, according to Lemmas 2.3 and 2.5 the set of zeros of $\left.f_{y}\right|_{\Gamma}$ consists of a single point $z_{1}$. Moreover, $|j-i|=\mu$, where $\mu$ is the order of $z_{1}$. Our objective is to prove that $\mu=1$. To do this we will point out a point $\widetilde{y} \in Y_{i j}$ such that $\left.f_{\widetilde{y}}\right|_{\Gamma}$ has simple zeros only.

According to hypothesis (5) of the theorem for $v=v(y) \in \mathbb{C}^{n}$ there exists a positive $\epsilon$ such that for any $c \in \mathbb{R},|c|<\epsilon$, and any $z \in \mathbb{D}_{r}, r:=\min \left\{\rho, \frac{\delta}{2 M}\right\}$, we have

$$
\begin{gathered}
f_{y+c v}(z)=f_{y}(z)+c g_{y+c v}(z), \text { where } \\
\left|g_{y+c v}(0)\right| \geq \frac{5 \delta}{6} \quad \text { and }\left|g_{y+c v}(z)-g_{y+c v}(0)\right| \leq \frac{3 \delta}{4} .
\end{gathered}
$$

This means that $g_{y+c v}$ is non-zero in $\mathbb{D}_{r}$. (Recall also that $\Gamma \subset \mathbb{D}_{r}$.) If $\epsilon$ is sufficiently small, there exists the continuous map $\beta_{y}: I_{\epsilon} \rightarrow B$ defined by

$$
f_{\beta_{y}(t)}(z)=f_{y}(t z) .
$$

Here $I_{\epsilon}=\{t \in \mathbb{R}:|t-1|<\epsilon\}$. Then, as in the proof of Lemma 2.3, we have, say,

$$
\beta_{y}(1+\epsilon) \in Z_{j} \quad \text { and } \quad \beta_{y}(1-\epsilon) \in Z_{i} .
$$

In particular, there is a positive $\epsilon^{\prime} \leq \epsilon$ such that for each real $c,|c|<\epsilon^{\prime}$, the continuous map $\beta_{y, c}: I_{\epsilon} \rightarrow B$,

$$
f_{\beta_{y, c}(t)}(z)=f_{y+c v}(t z),
$$

is correctly defined and $\beta_{y, c}(1+\epsilon) \in Z_{j}, \beta_{y, c}(1-\epsilon) \in Z_{i}$. Thus we can find an $s=s(c) \in I_{\epsilon}$ such that $\beta_{y, c}(s) \in Y_{i j}$, i.e. the number of zeros of $\left.f_{\beta_{y, c}(s)}\right|_{\Gamma}$ equals $|j-i|:=\mu$. Now, from (3.1) by the Rouché theorem we obtain that for a sufficiently small $\epsilon$ the zeros of each function $\left.f_{\beta_{y, c}(t)}\right|_{\Gamma}, t \in I_{\epsilon}$, are situated in a small open disk $O \subset \mathbb{D}_{r}$ centered at $z_{1}$ such that in $O$ we have $f_{y}(z)=h(z)\left(z-z_{1}\right)^{\mu}$ with $h$ holomorphic and nowhere zero in a bigger open disk $O_{1} \supset \bar{O}$. Thus for zeros $w$ of $\left.f_{\beta_{y, c}(s)}\right|_{\Gamma}$ from (3.1) we obtain

$$
\frac{f_{y+c v}(s w)}{g_{y+c v}(s w)}=\frac{h(s w)}{g_{y+c v}(s w)}\left(s w-z_{1}\right)^{\mu}+c=0 .
$$

If we choose $c \neq 0$ in the above identity, we get that all zeros of $\left.f_{\beta_{y, c}(s)}\right|_{\Gamma}$ are simple. Thus for the point $\widetilde{y}:=\beta_{y, c}(s) \in Y_{i j}, c \neq 0$, we have $|j-i|=1$.

This completes the proof of the theorem.

3.2. Proof of Theorem 1.10, Let $P_{\lambda}(z)$ be the Poincaré map of (1.4). Clearly, it suffices to prove the theorem for the family $\mathcal{F}=\left\{f_{\lambda}\right\}, f_{\lambda}(z):=\frac{P_{\lambda}(z)}{z}-1$.

It is easy to see that $P_{\lambda}(t z)=t \cdot P_{\tilde{\lambda}}(z)$ for any $t \in \mathbb{D} \backslash\{0\}$, where $\tilde{\lambda}$ is the collection of coefficients $\widetilde{a}_{k i}, \widetilde{b}_{k i}$ defined by

$$
\widetilde{a}_{k i}=a_{k i} t^{k+i-1}, \quad \widetilde{b}_{k i}=b_{k i} t^{k+i-1}, \quad 1 \leq i+k \leq d .
$$


Therefore $\mathcal{F}$ is locally stable with respect to the family $T_{3}$ on each compact set where all $P_{\lambda}$ are defined. For instance, if $\lambda \in B_{c}(0, N), N:=\frac{1}{40 \pi \sqrt{d}}$, we can take the closed disk $\overline{\mathbb{D}}_{3 / 4}$ as such a set, see [Br2, Section 3]. Then by definition we have

$$
f_{\lambda}(z)=\sum_{i=0}^{\infty} c_{i}(\lambda) z^{i}, \quad \lambda \in B_{c}(0, N), \quad z \in \mathbb{D}_{3 / 4}
$$

where

$$
c_{0}(\lambda)=\exp \left\{\int_{0}^{2 \pi} \frac{a_{10} \cos ^{2} \phi+b_{01} \sin ^{2} \phi+\left(a_{01}+b_{10}\right) \sin \phi \cdot \cos \phi}{1+b_{10} \cos ^{2} \phi-a_{01} \sin ^{2} \phi+\left(b_{01}-a_{10}\right) \sin \phi \cdot \cos \phi} d \phi\right\}-1 .
$$

Moreover, let $\tilde{\lambda} \in \mathbb{C}^{d(d+3)}$ be such that all the $a_{k i}$ and $b_{k i}$ are zeros if $(k, i) \neq(0,1)$ or $(k, i) \neq(1,0)$. Then $f_{\widetilde{\lambda}}(z)=c_{0}(\widetilde{\lambda})$. If in the definition of $\widetilde{\lambda}$ we set, in addition, $a_{10}=b_{10}=N$ and $a_{01}=b_{01}$, then in this case we get $f_{\widetilde{\lambda}}(z)=e^{\frac{1}{20 \sqrt{d}}}-1 \neq 0$. This means that the family $\mathcal{F}$ satisfies condition (1.2).

Next, consider the vector $v \in \mathbb{C}^{d(d+3)}$ which consists of the coefficients $a_{k i}, b_{k i}$ such that

$$
a_{k i}=\left\{\begin{array}{ll}
0, & (k, i) \neq(1,0), \\
1, & (k, i)=(1,0),
\end{array} \quad b_{k i}=\left\{\begin{array}{cc}
0, & (k, i) \neq(0,1), \\
1, & (k, i)=(0,1) .
\end{array}\right.\right.
$$

We first estimate $\left|D_{v} f_{\lambda}(0)\right|$. By the definition for $\lambda \in B_{c}(0, N), d \geq 2$, we have

$$
\begin{gathered}
\left|D_{v} f_{\lambda}(0)\right|:=\left|\lim _{t \rightarrow 0} \frac{c_{0}(\lambda+t v)-c_{0}(\lambda)}{t}\right| \\
=\left|\exp \left\{\int_{0}^{2 \pi} \frac{a_{10} \cos ^{2} \phi+b_{01} \sin ^{2} \phi+\left(a_{01}+b_{10}\right) \sin \phi \cdot \cos \phi}{1+b_{10} \cos ^{2} \phi-a_{01} \sin ^{2} \phi+\left(b_{01}-a_{10}\right) \sin \phi \cdot \cos \phi} d \phi\right\}\right| \\
\times\left|\int_{0}^{2 \pi} \frac{1}{1+b_{10} \cos ^{2} \phi-a_{01} \sin ^{2} \phi+\left(b_{01}-a_{10}\right) \sin \phi \cdot \cos \phi} d \phi\right| \\
\geq\left(1-\frac{2 \pi N}{1-N}\right) \cdot\left(2 \pi-\frac{2 \pi N}{1-N}\right)>6:=\delta .
\end{gathered}
$$

We used here the inequalities

$$
\left|e^{s}\right| \geq 1-\frac{|s|}{1-|s|}
$$

and

$$
\left|\int_{0}^{2 \pi} \frac{1}{1+g(\phi)} d \phi\right| \geq 2 \pi-\sum_{j=1}^{\infty} \int_{0}^{2 \pi}|g(\phi)|^{j} d \phi \geq 2 \pi \cdot\left(1-\frac{M}{1-M}\right),
$$

valid for $|s|<1$ and $|g(\phi)| \leq M<1, \phi \in[0,2 \pi]$.

Now we estimate $\left|D_{v} f_{\lambda}(z)-D_{v} f_{\lambda}(0)\right|, \lambda \in B_{c}(0, N)$. We will use an equation for solutions of (1.5):

$$
r(\phi, \lambda, z)=z \cdot \exp \left\{\int_{0}^{\phi} H(\lambda, r(s, \lambda, z), s) d s\right\}, \quad H(\lambda, r, s):=\frac{P(r, s)}{1+Q(r, s)} .
$$


Also we use the following inequality (see [Br2, Section 3])

$$
|H(\lambda, r, s)| \leq \frac{N \sqrt{d}}{1-N \sqrt{d}}:=\delta_{N}, \quad \lambda \in B_{c}(0, N), r \in \mathbb{D}_{1}, s \in[0,2 \pi] .
$$

From the inequality

$$
|r(\phi, \lambda, z)| \leq|z| e^{2 \pi \delta_{n}} \leq|z| \cdot \frac{53}{50},
$$

valid while $|r(\phi, \lambda, z)|<1$, it follows that $r$ is well defined for $z \in \mathbb{D}_{50 / 53}$. Also, if $z \in \mathbb{D}_{3 / 4}$ we obtain from the last inequality that $|r(\phi, \lambda, z)|<\frac{4}{5}$. Now from (3.3) according to the Cauchy inequalities for derivatives of holomorphic functions it follows that

$$
\left|\frac{\partial H(\lambda, r, s)}{\partial r}\right| \leq 5 \delta_{N}, \quad \lambda \in B_{c}(0, N), r \in \mathbb{D}_{4 / 5}, s \in[0,2 \pi] .
$$

Finally, we will use the following identity, whose verification is straightforward:

$$
D_{v} H(\lambda, r, s)=\frac{1}{1+Q(r, s)}, \quad \text { where } \quad\left|\frac{1}{1+Q(r, s)}\right| \leq \frac{1}{1-N \sqrt{d}}:=\gamma_{n} .
$$

Combining together all previous inequalities, we get from (3.2)

$$
\begin{aligned}
& \sup _{\phi \in[0,2 \pi]}\left|D_{v}\left(\frac{r(\phi, \lambda, z)}{z}-1\right)\right| \leq \exp \left\{\int_{0}^{2 \pi}|H(\lambda, r(s, \lambda, z), s)| d s\right\} \\
\times & \int_{0}^{2 \pi}\left|D_{v} H(\lambda, r(s, \lambda, z), s)+\frac{\partial H(\lambda, r(s, \lambda, z), s)}{\partial r} \cdot D_{v} r(s, \lambda, z)\right| d s \\
\leq & e^{2 \pi \delta_{N}} \cdot\left(2 \pi \gamma_{n}+\frac{15 \pi}{2} \delta_{n} \cdot\left(\sup _{\phi \in[0,2 \pi]}\left|D_{v}\left(\frac{r(\phi, \lambda, z)}{z}-1\right)\right|\right)\right) .
\end{aligned}
$$

From here we obtain

$$
\sup _{\phi \in[0,2 \pi]}\left|D_{v}\left(\frac{r(\phi, \lambda, z)}{z}-1\right)\right| \leq \frac{4 \pi \gamma_{n} \cdot e^{2 \pi \delta_{N}}}{2-15 \pi \delta_{n} \cdot e^{2 \pi \delta_{n}}}<\frac{42}{5}, \quad \lambda \in B_{c}(0, N), z \in \mathbb{D}_{3 / 4} .
$$

In particular, since $r(2 \pi, \lambda, z):=P_{\lambda}(z)$, we obtain

$$
\left|D_{v} f_{\lambda}(z)\right|<\frac{42}{5} \text {. }
$$

From here and the Schwarz lemma we get for $z \in \mathbb{D}_{3 / 4}, \lambda \in B_{c}(0, N)$,

$$
\left|D_{v} f_{\lambda}(z)-D_{v} f_{\lambda}(0)\right| \leq \frac{4}{3} \cdot \frac{84}{5} \cdot|z|=\frac{112}{5} \cdot|z|:=M \cdot|z| .
$$

In particular, we have

$$
\frac{\delta}{2 M}=\frac{30}{224}>\frac{1}{8}:=r .
$$

Then, according to Theorem 1.7, for any non-singular domain $R \subset \mathbb{D}_{1 / 8}$ satisfying the hypotheses of Theorem 1.5 the graph of zeros $\mathcal{G}_{\mathcal{F}, R}\left(B_{c}(0, N)\right)$ of $\mathcal{F}$ in $B_{c}(0, N)$ is of $A$-type.

The proof of the theorem is complete. 


\section{REFERENCES}

[B] A. Baker, Transcendental Number Theory, Cambridge U. Press, 1975. MR 54:10163

$[\mathrm{BM}]$ E. Bierstone and P. D. Milman, Semianalytic and subanalytic sets, Inst. Hautes Études Sci. Publ. Math., No. 67 (1988), 5-42. MR89k:32011

[Br1] A. Brudnyi, On local behavior of analytic functions, J. of Funct. Analysis, 169 (1999), no. 2, 481-493. MR2001b:32008

[Br2] A. Brudnyi, Small amplitude limit cycles and the distribution of zeros of families of analytic functions. Ann. of Math. 154 (2001), 227-243. MR2002k:30051

[H] H. Hironaka, Subanalytic sets, Number Theory, Algebraic Geometry and Commutative Algebra, Kinokuniya, Tokyo, 1973, 453-493. MR 51:13275

[Ha] R. M. Hardt, Triangulation of subanalytic sets and proper light subanalytic maps, Invent. Math., 38 (1977), 207-217. Mr.56:12302

[I] Yu. Ilyashenko, Centennial history of Hilbert's 16th problem, Bull. Amer. Math. Soc. 39 (2002), no. 3, 301-354. MR2003c:34001

[RY] N. Roytvarf and Y. Yomdin, Bernstein classes, Ann. Inst. Fourier, 47 (1997), 825-858. MR.98h:34009a

Department of Mathematics and Statistics, University of Calgary, Calgary, AlBerta, Canada T2N 1N4 